\title{
Fiabilidad y validez de los diferentes sistemas de registros de la inclinación de la trayectoria condilar (ITC). Revisión bibliográfica
}

\author{
Ventura de la Torre J*, Domínguez Cardoso P**, Cañadas Rodríguez D***, \\ Jiménez-Castellanos Ballesteros $\mathrm{E}^{* * * * *}$
}

\section{RESUMEN}

Desde que fuera descrita por Balkwill, la inclinación de la trayectoria condilar ha representado el más importante parámetro a individualizar para realizar los trabajos de rehabilitación protésica. Son muchos los sistemas que se han desarrollado para determinar el valor de éste ángulo en nuestros pacientes. Registros plásticos, dinámicos, gráficos... Es nuestra intención por ello, realizar una revisión a cerca de la fiabilidad y la validez de todos estos procedimientos, que por norma general han resultado controvertidos a lo largo del tiempo y de los que existe poco consenso sobre la exactitud de sus mediciones.

Palabras clave: Inclinación de la trayectoria condilar, Pantografía, Axiografía, Registros interoclusales.

\section{SUMMARY}

Since it was described by Balkwill in 1866, the protrusive condylar inclination has represented the most important parameter that we must know in order to make an oral rehabilitation. There are a lot of systems developed to determine this agle in our patients: interocclusal estatic or dynamic records, graphics... We intend for that reason to get a revision about the reliability and validity of these procedures, that commonly have been considered controversial and with little consensus about the accuracy of its measurements.

Key words: Protrusive condylar inclination, Axiography, Pantography interocclusal records.

Fecha de recepción: Octubre 2006.

Aceptado para publicación: Diciembre 2006.

Unidad Docente de Prótesis Estomatológica y Oclusión. Facultad de Odontología. Universidad de Sevilla.

* Colaborador Clínico.

** Profesor Asociado.

*** Profesor Titular.

**** Catedrático.

Ventura de la Torre J, Domínguez Cardoso P, Cañadas Rodríguez D, Jiménez-Castellanos Ballesteros E. Fiabilidad y validez de los diferentes sistemas de registro de la inclinación de la trayectoria condilar (ITC). Revisión bibliográfica. Av. Odontoestomatol 2007; 23 (2): 83-90.

\section{INTRODUCCIÓN}

El 4 de Junio de 1866, Francis H. Balkwill (1) realizó una presentación ante la Sociedad Odontológica de
Gran Bretaña sobre los movimientos maxilo-mandibulares y sus relaciones entre ellos. Sus descripciones fueron sorprendentes para la época pero consideró imposible realizar la medición de la inclinación 
de la trayectoria condilar (ITC) en el sujeto vivo. Aun así presentó un instrumento para medir "el ángulo formado entre el plano que delimitan dos líneas trazadas desde los cóndilos al punto incisal y el plano oclusal". Estimó este ángulo en un valor medio de unos $26^{\circ}$ y fue denominado "ángulo de Balkwill”.

Dos años antes, William G.A. Bonwill (2) realizó una descripción del movimiento hacia adelante y abajo de los cóndilos, pero no reconoció la influencia de este fenómeno en la articulación dentaria, y no hizo intentos de determinar estos parámetros.

Charles E. Luce, de Harvard (3), fue probablemente el primero en utilizar un arco facial mandibular, en el año 1989, para reproducir los movimientos mandibulares, y específicamente el trayecto condilar. Aplicó un método fotográfico, precursor de los sistemas optoelectrónicos actuales, sugiriendo que el trayecto condilar era curvo, y que presentaba una gran variabilidad individual.

En 1896, William E. Walker (4) diseñó el primer articulador que permitía realizar ajustes individuales de la ITC ("Articulador fisiológico de Walker"). Para realizar este ajuste diseñó un aparato llamado "Clinómetro facial", nunca patentado, pionero de los sistemas de registro extraoral. Posteriormente realizó una modificación del procedimiento de Luce, pudiendo considerarlo como el predecesor de los axiógrafos actuales.

Entre 1901 y 1905, Carl Christensen (4), de Copenhagen, transmitió sus observaciones acerca del espacio de separación posterior que ocurre entre las arcadas maxilar y mandibular cuando se realiza un movimiento protrusivo e introdujo un articulador similar al de Walker pero más sencillo. Sugirió utilizar ceras en protrusiva para regular la inclinación de la trayectoria condilar, pues observó, que el espacio de separación mencionado se correspondía proporcionalmente a dichas inclinaciones, describiendo así el denominado "Fenómeno de Christensen", término acuñado por Ulf Posselt (5), aunque realmente lo correcto sería otorgarle la denominación a Balkwill, pues fue éste quien primero lo describió en 1866.

En 1924 Beverly B. McCollum (6) de Los Angeles, desarrolló el primer método para localización del eje de Bisagra mediante una modificación del arco facial de Snow. Unos diez años más tarde junto con Charles E. Stuart, presentó un instrumento capaz de registrar los movimientos mandibulares en los tres planos del espacio, el "Gnathograph", mejorado en una segunda versión que apareció en 1935 y presentado de forma definitiva en 1937. Nacía así el primer pantógrafo mecánico tal y como lo entendemos hoy en día. Stuart, también en solitario patentó su propio pantógrafo y articulador en el año 1955.

Registros plásticos posicionales y dinámicos, gráficos, axiógrafos y pantógrafos mecánicos o electrónicos... Son muchos los sistemas de registro de la dinámica condilar que se han ido desarrollando con el paso del tiempo, y también es mucha la controversia que podemos encontrar en la literatura sobre la aceptación de cada uno de ellos.

\section{INFLUENCIA DE LA ITC EN LA MORFOLOGÍA OCLUSAL}

Se puede considerar a la ITC un determinante de la altura de las cúspides y de la profundidad de la fosa. La relación del plano de oclusión con la inclinación del trayecto condíleo, influye de tal modo, que a mayor divergencia entre el plano de oclusión y el ángulo de la eminencia mayor podrá ser la altura de las cúspides y más profundas las fosas y viceversa.

Weimberg (7) apunta que un error de $5^{\circ}$ en la inclinación de la trayectoria condilar en el movimiento de no trabajo, causa un error de $0,5 \mathrm{~mm}$ en la altura de la cúspide de no trabajo de un segundo molar (en una cúspide de $3 \mathrm{~mm}$ de altura), aunque sin influencia en la cúspide de trabajo. Price (8), estudia el efecto de los cambios de ajuste condilar en el articulador sobre la cúspide mesiolingual del primer molar superior durante los movimientos laterales y protrusivos, y determina que las modificaciones en la ITC afectan a la altura de la cúspide y a la posición de los surcos. Si la ITC se eleva, la altura de la cúspide mandibular de no trabajo se incrementa, sin afectar al lado de trabajo, mientras que la posición de la cresta y el surco se mueve mesialmente en la excursión de no trabajo. Además, la ITC es el único parámetro que afecta a la altura de la cúspide en el movimiento protrusivo. 
Ventura de la Torre J, Domínguez Cardoso P, Cañadas Rodríguez D, Jiménez-Castellanos Ballesteros E Fiabilidad y validez de los diferentes sistemas de registro de la inclinación de la trayectoria condilar (ITC). Revisión bibliográfica

\section{PROCEDIMIENTOS DE REGISTRO. ESTUDIOS DE PRECISION Y VALIDEZ}

Son muchos los estudios que con anterioridad han pretendido determinar en unos casos la fiabilidad, en otros la validez de los diferentes sistemas y procedimientos para el cálculo de los determinantes posteriores y su programación en el articulador. Nos centraremos principalmente en los relacionados con los sistemas axiográficos-pantográficos, por estar estos en continua evolución y haber incorporado recientemente los últimos avances en electrónica e informática con la finalidad de mejorar la fiabilidad de estos sistemas y la comodidad de aplicación para el clínico.

El último de los sistemas que ha hecho aparición, es el Arcus Digma (KaVo elektrotechnisches, Werk, GMBH, Leutkirch, Germany). Tan solo Pröschel (9) y cols. presentan un estudio sobre el Arcus Digma en el año 2002, consistente en la determinación de la precisión del mismo sobre un articulador con valores predeterminados. La mayor discrepancia obtenida fue de $1,5^{\circ}$. No obstante, entienden que se trata de un estudio sobre articulador y que en mediciones reales sobre pacientes se le presupone al sistema errores de entre dos y tres grados de diferencia con el valor real.

Este mismo sistema de medición sobre articuladores con valores preajustados ha sido utilizado en otras ocasiones con diversos propósitos.

Celar y Tamaki (10) investigan la precisión de un pantógrafo electrónico, el Cadiax Compact (Gamma Dental Software, Klosterneuburg, Austria) en la determinación del ángulo de Bennet y la ITC sobre un articulador Artex, preajustado con valores de ITC de $20^{\circ}, 40^{\circ}$ y $60^{\circ}$ que eran calibrados con un digitalizador tridimensional, obtuvieron diferencias que oscilaban entre los $0^{\circ}$ y los $6^{\circ}$, con una media de $1-2^{\circ}$, no existiendo diferencias significativas entre las mediciones del Cadiax Compact y el ajuste de $40^{\circ}$ de ITC a los 3 y a los $5 \mathrm{~mm}$, pero sí para el resto de mediciones $(p<0,01)$ lo cual puede considerarse clínicamente aceptable puesto que ningún sistema de programación de un articulador ha demostrado una precisión mayor.

Anderson (11) evalúa la precisión y la fiabilidad del pantógrafo electrónico Denar Pantronic realizando las mediciones sobre el articulador Denar D5-A. El pantógrafo midió la ITC de $25^{\circ}$ con errores considerados como aceptables de entre 2 y $5^{\circ}$, pero no aclaran si realizaban una calibración previa para verificar los ajustes del articulador.

Chang y cols (12). También con el Cadiax Compact realizan las mediciones sobre cinco articuladores diferentes, justificando el método, por no existir ningún procedimiento exacto para conocer los valores reales de la ITC real del individuo y por tanto no ser factible una comparación. Encuentra que las menores desviaciones de las mediciones se producen a los $10 \mathrm{~mm}$, por lo que recomienda el ajuste de la ITC en esta posición, aunque consideran que esto exige al paciente realizar un movimiento mandibular exagerado y poco natural.

También Pelletier (13) realiza un estudio sobre articuladores, empleando 9 diferentes sistemas de medida de la ITC (registros plásticos posicionales y gráficos con pantografía mecánica, electrónica y analizadores simplificados). Tras el análisis y comparación de los resultados determinan que ninguno de los materiales para registro interoclusal resultó ser preciso ni reproducible. El pantógrafo electrónico, por su parte, (Pantronic) obtuvo resultados más precisos y reproducibles que el pantógrafo mecánico y fueron, junto con dos de los axiógrafos (Whip-Mix y Denar), los sistemas más precisos y fiables en la medición de la ITC aunque, entre todos ellos, fue el Pantronic el que obtuvo mejores resultados.

Otros autores han preferido sin embargo realizar este tipo de estudio sobre pacientes, por considerar que las mediciones realizadas sobre los articuladores falsean los resultados al eliminar los factores propios del individuo. En la mayoría de los casos utilizan dos o más sistemas de medición sobre el mismo individuo para realizar así una comparativa y compensar la falta de un "Gold Standard".

Petrie y cols (14) realizan una comparación de las mediciones obtenidas de manera repetida sobre diez pacientes entre un pantógrafo mecánico y otro electrónico (Denar y Cadiax Compact respectivamente), encontrando una escasa reproducibilidad con el pantógrafo electrónico y atribuye la variación al 
aparato. Una posible explicación es la interferencia en los movimientos de la cubeta mandibular y el material de fijación con la que se ajusta el arco inferior a la arcada, la falta de un puntero central único puede justificar la inconsistencia de los resultados en el tiempo y por todo ello aconseja prudencia ante los buenos resultados de los estudios in vitro.

Wessling (15) realiza una comparación directa entre el Cadiax-System de la empresa Gamma y el precursor del Arcus Digma, el Jaw Motion Analyzer de la empresa Zebris. Encontrando un valor de ICC (intraclass correlation) superior a 0,80 y concluyendo que las diferencias entre uno y otro sistema no resultaron relevantes clínicamente.

Wagner y cols (16) comparan el Cadiax-System de Gamma, y un sistema optoelectrónico sin cables, consistente en la captación y registro del movimiento mandibular mediante unos digitalizadores infrarrojos (Polaris) combinados con sensores tridimensionales. Los sensores (dos), son posicionados para el estudio en cada uno de los dos arcos que conforman el Cadiax-System en una posición arbitraria, observando una alta correlación entre los registros de ambos sistemas, con una ICC en la medición de la ITC de entre 0,86 y 0,95 .

Bernhardt y cols (17) pretenden medir la precisión del Cadiax Compact System mediante su aplicación sobre 30 sujetos sanos, y a la vez determinar si existen diferencias relevantes entre la utilización del centro real de rotación condilar, o uno promedio. Encontró una alta concordancia entre las inclinaciones y trayectos condilares (ICC $>0,80$ ) registrados por el Cadiax Compact en los diferentes días independientemente de que fueran medidos en centro real o promedio.

Forcén y cols (18) realizan un estudio para determinar la fiabilidad y validez del axiógrafo Axio-Quick (FAG Dentaire) empleado por tres operadores distintos sobre los mismos sujetos para medir la ITC a los $5 \mathrm{~mm}$ de desplazamiento protrusivo. Encontrando discrepancias no significativas de un promedio de 1,8 grados sexagesimales. El ICC promedio fue 0,87 por lo que concluían que es un procedimiento muy fiable.
En otro estudio realizado (19) con el Axio-Quick con registro protrusivo a los 2, 4, 5 y $6 \mathrm{~mm}$ no encontraron tampoco diferencias significativas.

Jiménez-Castellanos y Domínguez (20) realizan un estudio con el Axio-Quick comparando los valores de la ITC obtenidos mediante los movimientos de apertura y protrusivo. Encontrando valores muy similares ya que según sus resultados hasta los $5 \mathrm{~mm}$ de movimiento las trayectorias prácticamente se superponían.

Esteban (21) realiza un estudio de los trazados obtenidos también con el Axio-Quick en pacientes sin patología y con disfunción leve o moderada del aparato estomatognático. Se midió la ITC a los 3, 5, 7 y $9 \mathrm{~mm}$ de desplazamiento en movimientos protrusivos, de apertura y lateralidad de no trabajo, concluyendo tras el análisis estadístico que en los pacientes normales el valor de la ITC entre los tres tipos de movimientos no mostraba diferencias significativas en los 7 primeros milímetros de desplazamiento, produciéndose una disminución progresiva de dicho valor desde los 3 a los $9 \mathrm{~mm}$ de desplazamiento. Las mayores desviaciones en los pacientes normales se registraron a los $3 \mathrm{~mm}$ en las tres clases de movimientos.

Morneburg y Proschel (22), por contra, aconsejan utilizar el movimiento protrusivo y no el de apertura para la regulación del articulador. Realizaron un estudio sobre sujetos sin patología utilizando 10 puntos distintos de partida para registrar los trayectos condilares durante los movimientos de protrusión, lateralidad y apertura y cierre. En protrusión, los trazados de los distintos puntos de partida eran iguales independientemente de la localización del punto monitorizado, mientras que en la apertura y cierre diferían considerablemente mostrando incluso irregularidades propias de pacientes que pudieran tener desordenes temporomandibulares. Las variaciones e irregularidades eran menores en los casos del centro real de rotación condilar partiendo de relación céntrica o de máxima intercuspidación, lo que coincide con lo resultados de Naeije y cols (23) que realizan de 14 a 18 mediciones con un axiógrafo optoelectrónico sobre cuatro pacientes asintomáticos partiendo del centro real de rotación condilar y de otros cuatro puntos cercanos a éste 
separados no más de $10 \mathrm{~mm}$, formando las cuatro esquinas de un cuadrado con centro en el CRRC. Los trazados de dicho centro mostraron las menores variaciones en comparación con los otros cuatro puntos.

Igualmente, Peck y cols $(24,25)$ realizan un estudio sobre los movimientos condilares de 5 puntos condilares distintos. Fueron registrados y computados en los planos sagital y horizontal con un sistema optoelectrónico (Jaws 3D, Metropoly AG, Zurich, Switzerland). En un primer estudio sólo los movimientos de apertura y cierre fueron analizados, encontrando en todos los casos que las trayectorias de los cinco puntos eran diferentes en forma y dimensión de los demás. Posteriormente sumaron al estudio los movimientos de protrusión y lateralidad (lado de no trabajo), muy poca variabilidad fue encontrada entre las trayectorias de los diferentes puntos durante dichos movimientos, aunque sugiere que los movimientos protrusivos de mayor componente de "traslación" son más fáciles de registrar y reproducir que los movimientos con un mayor componente de rotación condilar.

Price (26) por su parte, realiza un estudio para determinar la repercusión en el ajuste del articulador al utilizar el eje terminal de bisagra o un eje arbitrario a la hora de tomar los registros gráficos con el pantógrafo electrónico Denar Pantronic concluyendo que no influye la consideración de uno u otro.

Los trabajos hasta ahora referidos están realizados sobre pacientes sin patología, existen, sin embargo, numerosos trabajos realizados en pacientes disfuncionales coincidiendo en general en sus conclusiones en que el sujeto libre de signos y síntomas de disfunción temporomandibular tiene la capacidad de repetir con una gran exactitud los movimientos mandibulares y sus trazados pantográficos por el contrario de los sujetos disfuncionales. De esto se deriva que el pantógrafo pueda ser usado como una herramienta de diagnóstico de los distintos grados de patología articular; no obstante algunos autores discrepan de esta afirmación (27).

Volviendo a la determinación de la fiabilidad de los procedimientos en el cálculo de la ITC, otros autores han optado por la comparación de los sistemas de registro gráfico con el clásico procedimiento de registros plásticos intraorales basados en el fenómeno de Christensen: Domínguez y Jiménez-Castellanos (28) comparan los resultados obtenidos para la ITC con registros plásticos posicionales y el axiógrafo Axio-Quick, observando mayor precisión en los resultados mediante los registros plásticos así como una gran discrepancia promedio de $21,5^{\circ}$ de diferencia entre uno y otro método que tratan de explicar por distintas hipótesis, como el promediado del valor de los registros plásticos en el articulador que disminuye las discrepancias de valor, el empleo de distintos planos de referencia para realizar las mediciones en ambos casos o el distinto desplazamiento realizado en uno u otro método en el que se realiza la determinación del valor de la ITC.

Del Río Highsmith y cols (29) realizan un estudio muy similar de comparación entre ambos sistemas (Axio-Quick y registros posicionales de cera). Encontrando, sin embargo, variaciones superiores en los registros posicionales.

Dos Santos Jr (30) compara los registros plásticos con el axiógrafo Whip-Mix PT quick-set recorder obteniendo también valores superiores estadísticamente significativos con el axiógrafo que presentó además una mayor precisión.

Ecker y cols (31) igualmente comparan los registros de cera y dos axiógrafos: el Whip-Mix quick set recorder y el Panadent quick analyzer recorder. Nuevamente encontraron que los registros plásticos conseguían lecturas inferiores con un mayor rango de valores. El Whip-Mix a su vez valores mayores que el Panadent. Ecker explica la discrepancia argumentando que ambos sistemas utilizan diferentes planos anteroposteriores, lo que puede justificar las diferencias de los resultados.

Peraire y cols (32) presentan un estudio de los factores que pueden alterar la exactitud y la precisión de los registros de cera en el cálculo de la ITC, operador, tipo de articulador, etc.

Existen otros estudios a cerca de los factores que modifican e influyen en la determinación de la ITC mediante los registros plásticos posicionales. Domín- 
guez y Borrero (33) estudian la relación existente entre el desplazamiento condilar y el dentario en el movimiento protrusivo, concluyendo que el desplazamiento condilar será de distinta magnitud en función de la profundidad de la ITC, lo cual puede justificar la diferencia de valor obtenida con registros gráficos y posicionales.

Forcén y cols (34) realizan un estudio para determinar si existen diferencias significativas en el valor de la ITC registrado mediante ceras a los 4,5 y $6 \mathrm{~mm}$ de movimiento protrusivo medidos a nivel condilar. Los resultados por ellos obtenidos indicaban que sólo existía una diferencia significativa entre los 5 y los $6 \mathrm{~mm}$ para el lado izquierdo y casi significativa entre los 4 y los $6 \mathrm{~mm}$ también para el lado izquierdo, ascendiendo aproximadamente a dos grados.

\section{CONCLUSIÓN}

De los trabajos analizados podemos deducir que si bien la mayoría de los métodos empleados presentan una alta precisión al conseguir una gran tasa de reproducibilidad de resultados, sin embargo, existen discrepancias en los valores obtenidos con los distintos procedimientos de registro gráficos y posicionales, no suficientemente explicados.

Por otra parte la inexistencia de un patrón oro (gold standard) de referencia que determine "el valor real de la ITC", hace imposible precisar la validez de los distintos procedimientos in vivo, siendo necesarios estudios complementarios que aclaren estas discrepancias.

\section{BIBLIOGRAFÍA}

1. Balkwill FH. The best form and arrangement of artificial teeth for mastication. Trans Odont Soc Great Britain 1866;5:133-58.

2. Bonwill WGA. Articulation and articulators. Trans Am Dent Assoc 1864;4:76-9.

3. Luce CE. The movements of the lower jaw. Boston Med Surg J 1889;121:8-11.
4. Starcke EN. The History of articulators: early attempts to reproduce mandibular movements, partIII: searching for the solution to a puzzle. J Prosthodont 2000;9:217-22.

5. Posselt U. Physiology of Occlusion and Rehabilitation. Philadelphia, PA, F.A: Davis Co: 1962.

6. Starcke EN. The History of articulators: from facebows to the Gnatograph. A brief history of early devieces developed for recordings condylar movement. Part II. J Prosthodont 2002; 11:53-62.

7. Weimberg LA. An evaluation of basic articulators and their concepts. Part II: arbitrary, positional, semiadjustable articulators. J Prosthet Dent 1965;15:826-46.

8. Price RB, Kolling JN, Clayton JA. Effects of changes in articulator settings on generated occlual tracings. Part I: condylar inclination and progressive side shift settings. J Prosthet Dent 1991;65:237-43.

9. Pröschel P, Morneburg P, Hugger A, Korda $\beta$ B, Ottl P, Niedermeier W, Wichmann M. Articulatorrelated registration. A simple concept for minimizing eccentric occlusal errors in the articulator. Int J Prosthodont 2002;15:289-94.

10. Celar AG, Tamaki K. Accuracy of recordings horizontal condylar inclination and Bennett angle with the Cadiax Compact. J Oral Rehabilitation 2002;29:1076-81.

11. Anderson GC, Schulte JK, Arnold TG. An in vitro study of an electronic pantograph. J Prosthet Dent 1987;57:577-80.

12. Chang WSW, Romberg E, Driscoll CF, Tabacco MJ. An in vitro evaluation of the reliability and validity of an electronic pantograph by testing with five different articulators. J Prosthet Dent 2004;92:83-9.

13. Pelletier LB, Campbell SD. Comparison of condylar control settings using three methods: 
Ventura de la Torre J, Domínguez Cardoso P, Cañadas Rodríguez D, Jiménez-Castellanos Ballesteros E

Fiabilidad y validez de los diferentes sistemas de registro de la inclinación de la trayectoria condilar (ITC). Revisión bibliográfica

a bench study. J Prosthet Dent 1991;66:193200.

14. Petrie CS, Woolsey GD, Williams K. Comparison of recordings obtained with computerized axiography and mechanical pantography at 2 time intervals. J Prosthodont 2003;12: 102-10.

15. Weßling F, Kordaß B, Schwahm B. Klinischer Vergleich der elektronischen Messysteme Gamma-Cadiax und JMA. Zahnärztl Welt 2000; 109:603-9.

16.Wagner A, Seemann R, Schicho K, Ewers R, Piehslinger E. A comparative analysis of optical and conventioanl axiography for the analysis of temporomandibular joint movements. J Prosthet Dent 2003;90:503-9.

17. Bernhardt O, Küppers N, Rosin M, Meyer G. Comparative test of arbitrary and kinematic transverse horizontal axis recordings of mandibular movements. J Prosthet Dent 2003;89: 175-9.

18. Forcén Báez A, Ruiz Navas MT, Serrano Belmonte I. Axiografía, un estudio sobre su fiabilidad y validez. Revista Europea de Odontoestomatología 2004;16:39-44.

19. Forcén Báez A, Ruiz Navas MT, Serrano Belmonte I, Royo-Villanova Pérez ML. Variaciones del ángulo de la pendiente condilar mediante registros axiográficos. Revista Europea de Odontoestomatología 2005; 17:27-34.

20. Jiménez-Castellanos E, Domínguez Fresco $M$. Comparación del valor de la inclinación de la trayectoria condilar mediante registros entre un movimiento de apertura y un movimiento protrusivo. Revista Europea de Odontoestomatología 1990;2:17-26.

21. Esteban Herrera I. Revisión experimental de los registros de las trayectorias condilares en el plano sagital [Tesis doctoral]. Sevilla: Universidad de Sevilla. 1995.

22. Morneburg T, Proschel PA. Differences between traces of adjacent condylar points and their impact on clinical evaluation of condyle motion. Int J Prosthodont 1998;11:317-24.

23. Naeije M, Huddleston Slater JJ, Lobbezoo F. Variation in movement traces of the kinematic center of the temporomandibular joint. J Orofac Pain 1999;13:121-7.

24. Peck CC, Murray GM, Johnson CWL, Klineberg IJ. The variability of condylar point pathways in open-close jaw movements. J Prosthet Dent 1997;77:394-404.

25. Peck CC, Murray GM, Johnson CWL, Klineberg IJ. Trajectories of condylar points during nonworking and protrusive movements of the mandible. J Prosthet Dent 1999; 82: 322-31.

26. Price RB, Gerrow JD, Ramier WC. Potential errors when using a computerized axiograph. J Prosthet Dent 1989;61:155-60.

27. Harper RP, Schneiderman E. Condylar movement and centric relation in patients with internale derangement of the temporomandibular joint. J Prosthet Dent 1996 75:67-71.

28. Domínguez Fresco M, Jiménez-Castellanos E. Valoración de la ITC mediante registros gráficos y plásticos. Estudio experimental sobre 25 casos. Revista Europea de Odontoestomatología 1989;1:365-70.

29. Del Río Highsmith J, López Lozano JF, Martínez Vázquez de Parga JA. Determinación de la trayectoria condílea mediante registro extraoral (sistema Axio-Quick), en comparación con registros intraorales en cera. Revista Europea de Odontoestomatología 1989;1:17-22.

30. Dos Santos Jr J, Nelson S, Nowlin T. Comparison of condylar guidance setting obtained from a wax record versus an extraoral tracing: a pilot study. $\mathrm{J}$ Prosthet Dent 2003 89:54-9.

31. Ecker GA, Goodacre CJ, Dykema RW. A comparison of condylar control settings obtained from wax interocclsal records and simplified mandibular motion analyzers. J Prosthet Dent 1984;51:404-6. 
32. Peraire Ardévol M, Martínez-Gomis J, Anglada Cantarell JM, Bizar Ramoneda J, Nogués Pujol L, Salsench Cabré J. Factores que alteran la exactitud y precisión en la determinación del ángulo de la guía condílea mediante registros de cera con un articulador semiajustable. Revista Europea de Odontoestomatología 2002; 14:257-62.

33. Domínguez Fresco M, Borrero Gallardo R. Relación entre desplazamiento condilar y dentario en un movimiento protrusivo. Estudio experimental sobre 40 casos. Revista europea de Odontoestomatología 1991;3:35-40.
34. Forcén Báez A, Ruiz Navas MT, Royo Villanova Pérez ML, Pérez Flores D. Estudio de las variaciones del ángulo de inclinación de la trayectoria condilar mediante registros posicionales. Revista europea de Odontoestomatología 2003;15:14150.

\section{CORRESPONDENCIA}

D. Javier Ventura de la Torre

C/ Recaredo $n^{\circ} 233^{\circ} \mathrm{C}$.

41003 Sevilla

E-mail: javiventura@yahoo.es 